\title{
Qualidade de vida de indivíduos com lesão medular praticantes de Basquetebol em cadeira de rodas
}

Fernanda dos Reis Rodrigues, Agege Haidar Filho, Adelzir Malheiros E S. C. B. Haidar, Karla Virgínia Bezerra de Castro Soares, Sarah Tarcisia Rebelo Ferreira de Carvalho 


\title{
Qualidade de vida de indivíduos com lesão medular praticantes de Basquetebol em cadeira de rodas
}

\section{Quality of life of individuals with spinal cord injury Basketball practitioners in wheelchair}

\begin{abstract}
Fernanda dos Reis Rodrigues ${ }^{1}$, Agege Haidar Filho ${ }^{2}$, Adelzir Malheiros E S. C. B. Haidar ${ }^{3}$, Karla Virgínia Bezerra de Castro Soares ${ }^{4}$ Sarah Tarcisia Rebelo Ferreira de Carvalho ${ }^{5}$
\end{abstract}

\section{Resumo}

Introdução: Lesão medular é uma injúria à medula espinhal que danifica a rede neural do indivíduo, causando alterações na coordenação motora e sensorial e no controle autônomo dos órgãos. Tais lesões podem ser irreversíveis, levando a consequências sociais e econômicas para o paciente, a família e a sociedade. Objetivo: Comparar a qualidade de vida de indivíduos com lesão medular praticantes de basquetebol em cadeira de rodas e de indivíduos com lesão medular não praticantes deste esporte. Material e método: Estudo comparativo e descritivo do tipo transversal, com abordagem quantitativa, com 20 cadeirantes do gênero masculino, portadores de lesão medular, sendo 10 praticantes de basquetebol e 10 não praticantes, com idade de 20 a 55 anos, da cidade de Marabá-PA. A qualidade de vida foi avaliada através da Versão Brasileira do SF-36. A análise dos dados foi realizada utilizando-se o programa SPSS 18.0 , considerando $p \leq 0,05$. Resultados: Os participantes lesados medulares que praticavam basquetebol em cadeira de rodas apresentaram escores significativamente superiores em relação aos aspectos referentes à capacidade funcional, aspectos físicos e vitalidade da qualidade de vida quando comparados de indivíduos com lesão medular não praticantes de basquetebol em cadeira de rodas. Conclusão: Considera-se que o basquetebol em cadeira de rodas pode ser uma importante estratégia terapêutica capaz de influenciar a qualidade de vida de seus praticantes.

Palavras-chave: Qualidade de vida; Lesão medular; Basquetebol em cadeira de rodas.

\footnotetext{
$1 \quad$ Graduado em Fisioterapia pela Universidade Ceuma.

2 Professor Especialista do Curso de Fisioterapia da Universidade Ceuma.

3 Professora Mestre do Curso de Fisioterapia da Universidade Ceuma.

4 Professor a Mestre do Curso de Fisioterapia da Universidade Ceuma.

5 Professora Doutora do Curso de Fisioterapia da Universidade Ceuma.
} 


\section{Abstract}

Introduction: Spinal cord injury is an injury to the spinal cord that damages the neural network of the individual, causing changes in motor and sensory coordination and autonomous control of the organs. Such lesions may be irreversible, leading to social and economic consequences for the patient, family and society. Objective: To compare the quality of life of individuals with spinal cord injury Basketball practitioners wheelchair users and individuals with spinal cord injury do not practice this sport. Methods: Comparative and descriptive crosssectional study with a quantitative approach, with 20 wheelchair males, patients with spinal cord injury, 10 practitioners basketball and 10 non-practitioners, aged 20-55 years old, the city of Maraba-PA. Quality of life was assessed by the Brazilian version of the SF-36. Data analysis was performed using SPSS 18.0, considering $p \leq 0.05$. Results: The spinal cord injured participants who practiced wheelchair basketball had significantly higher scores for aspects related to functional capacity, physical aspects and vitality of quality of life when compared to individuals with spinal cord injury not practicing basketball in a wheelchair. Conclusion: It is considered that the wheelchair basketball can be an important therapeutic strategy to influence the quality of life of its practitioners.

Keywords: Quality of life; Spinal cord injury; Wheelchair basketball.

\section{Introdução}

Portador de deficiência é aquele que apresenta em caráter temporário ou permanente, significativas diferenças físicas, sensoriais ou intelectuais, decorrentes de fatores inatos ou adquiridos, que acarretam dificuldades em sua interação com o meio social, necessitando por isso, de recursos especializados para desenvolver seu potencial e superar ou minimizar suas dificuldades (GORLA et al., 2007).

A lesão medular é uma injúria à medula espinhal que danifica a rede neural do indivíduo, danificando a transmissão, modificação coordenação motora e sensorial e o controle autônomo dos órgãos. Tais lesões podem ser irreversíveis, alterando de maneira drástica o custo de vida e levando a consequências sociais e econômicas para o paciente, a família e sociedade (NASCIMENTO; SILVA, 2007).

As lesões medulares são cada vez mais frequentes devido, principalmente, ao aumento da violência urbana. Os acidentes de trânsito e os ferimentos por arma de fogo são suas causas mais comuns. O traumatismo da medula pode resultar em alterações das funções motora, sensitiva e autônoma, implicando perda parcial e/ou total dos movimentos voluntários em membros inferiores (BAMPI; GUILHEM; LIMA, 2008). 
Segundo a Organização Mundial da Saúde (OMS), a definição de qualidade de vida é dada através da percepção em relação à sua posição da vida, no contexto da cultura e do sistema de valores nos quais vive e em relação a seus objetivos, expectativas padrões e preocupações. As condições funcionais e o nível de estresse são fatores que determinam a qualidade de vida do indivíduo (VALL; BRAGA; ALMEIDA, 2006).

$O$ basquete em cadeiras de rodas é um esporte praticado por indivíduos portadores de deficiência física permanente na parte inferior do corpo. Para atenuar as desigualdades durante as competições, em função das diversas possibilidades de deficiências, é utilizado um dispositivo adaptativo da cadeira de rodas (HORTA et al., 2009).

Ainda de acordo com Horta et al. (2009), o esporte permite a inclusão de portadores de deficiência, sem que haja discriminação. Tais aspectos fazem do basquete em cadeira de roda um dos esportes mais praticados entre atletas paralímpicos. Uma das consequências dessa popularização é a crescente busca da descrição e explicação dos diversos fenômenos desse esporte, na tentativa de uma melhor caracterização do mesmo e de seu praticante.

O esporte para paraplégicos e tetraplégicos consiste em uma atividade de educação para a saúde, uma vez que colabora para a inserção do indivíduo para a vida em sociedade ao mesmo tempo em que proporciona vivências de êxito, aumento da tolerância à frustração, contato social, além de tornar os indivíduos mais independentes, melhorar a autoimagem e a autoestima, desenvolver o potencial residual, melhorar a condição orgânica e funcional e aprimorar as qualidades físicas como a resistência, força e velocidade (MELLO et al., 2005).

Devido ao alto índice de violência e acidentes automobilísticos, existem um grande número de jovens com sequelas motoras e sensitivas, capazes de alterar e dificultar substancialmente a qualidade de vida e a funcionalidade desses indivíduos. Neste contexto, o presente estudo tem como objetivo comparar a qualidade de vida de indivíduos com lesão medular praticantes de basquetebol em cadeira de rodas e de indivíduos com lesão medular não praticantes deste esporte.

\section{Materiais e Métodos}

Trata-se de uma pesquisa comparativa e descritiva, do tipo transversal com abordagem quantitativa (THOMAS; NELSON; SILVERMAN, 2007).

A pesquisa foi realizada na cidade de Marabá-PA, no Ginásio Poliesportivo Hozorinho, localizado na Avenida Getúlio Vargas, no bairro da Marabá Pioneira, no período de outubro a novembro de 2012. 
A amostra foi composta por 20 indivíduos portadores de lesão medular, sendo todos cadeirantes do sexo masculino, com idade entre 20 e 55 anos, selecionados por amostragem não probabilística, divididos em dois distintos grupos, a saber: Grupo 1 (G1) compreendendo portadores de lesão medular que praticavam basquetebol em cadeira de rodas, três vezes por semana; e Grupo 2 (G2), com portadores de lesão medular não praticantes de basquetebol.

Os cadeirantes que aceitaram participar da pesquisa assinaram um termo de consentimento livre esclarecido, em pesquisa com seres humanos de acordo com a Resolução 466/2012, do Conselho Nacional de Saúde.

Os participantes de ambos os grupos $\mathrm{G} 1$ e $\mathrm{G} 2$ foram avaliados quanto à qualidade de vida através da versão Brasileira do questionário Medical Outcome Study-Mos Short From Health Survey (SF-36). Este questionário consiste em um instrumento genérico, traduzido para a língua portuguesa e validado, composto de 11 perguntas, incluindo 36 itens divididos em oito escalas: capacidade funcional (10 itens), aspectos físicos (04 itens), dor (02 itens), estado geral de saúde (05 itens), vitalidade (04 itens), aspectos sociais (02 itens), aspectos emocionais (03 itens) e saúde mental (05 itens). 0 escore em cada domínio é transformado em uma escala de 0 a 100, em que 100 indica a percepção do sujeito de melhor qualidade de vida relacionada à saúde nesse domínio. Consideram-se que essas dimensões representam valores humanos básicos relevantes na avaliação da qualidade de vida da população geral (CICONELLI, 1999; 2003).

A análise dos dados foi realizada por meio do programa SPSS 18.0. Para as análises descritivas, utilizou-se a distribuição de probabilidades (análise percentual), média e desvio padrão. A comparação dos resultados entre os grupos foi realizada mediante o teste Mann Whitney. Foram consideradas significantes as diferenças com $p \leq 0,05$.

\section{Resultados e discussão}

Participaram do estudo 20 indivíduos do sexo masculino, com média de idade de $38,1( \pm 9,4)$ no grupo dos praticantes de basquetebol em cadeira de rodas e 39,9 $( \pm 8,7)$ no grupo dos cadeirantes não praticantes deste esporte.

A tabela 1 apresenta a comparação da qualidade de vida de cadeirantes praticantes e não praticantes de basquetebol. 
Tabela 1 - Qualidade de vida de praticantes de basquetebol em cadeira de rodas e cadeirantes não praticantes de basquetebol da cidade de Marabá-PA, 2012.

\begin{tabular}{lccc}
\hline \multicolumn{1}{c}{ Domínios } & $\begin{array}{c}\text { Praticantes de } \\
\text { Basquetebol em } \\
\text { Cadeira de Rodas } \\
(\mathbf{n = 1 0 )}\end{array}$ & $\begin{array}{c}\text { Não praticantes de } \\
\text { Basquetebol em } \\
\text { Cadeira de Rodas } \\
(\mathbf{n = 1 0 )}\end{array}$ & $\mathbf{p}$ \\
\hline Capacidade funcional & 14,1 & 6,8 & $0,00^{*}$ \\
Aspectos Físicos & 12,9 & 8,0 & $0,05^{*}$ \\
Dor & 10,6 & 9,3 & 0,37 \\
Estado geral de Saúde & 12,3 & 8,6 & 0,15 \\
Vitalidade & 13,3 & 7,6 & $0,02^{*}$ \\
Aspectos sociais & 11,8 & 9,2 & 0,28 \\
Aspectos emocionais & 10,5 & 10,4 & 0,96 \\
Saúde mental & 10,6 & 10,4 & 0,93 \\
\hline
\end{tabular}

*Diferença estatisticamente significativa

Com relação à análise da qualidade de vida, verificou-se que os indivíduos que praticam basquetebol em cadeira de rodas apresentaram valores significativamente maiores quando comparados com os não praticantes deste esporte, em relação aos domínios capacidade funcional, aspectos físicos e vitalidade.

O domínio da capacidade funcional avalia a presença das limitações físicas e como ela interfere na capacidade física dos indivíduos. Os aspectos físicos avaliam as limitações na forma e quantidade de trabalho e o domínio vitalidade, por sua vez, é avaliado pelo nível de energia e fadiga de cada indivíduo (CICONELLI, 1999; 2003).

O estudo de Noce, Simim e Mello (2009) comparou a percepção da qualidade de vida de indivíduos com deficiência física sedentários e atletas de basquetebol em cadeira de rodas e constatou que o grupo ativo apresentou escores mais elevados em todas as dimensões (física, psicológica, social e ambiental).

Já a pesquisa de Orsi et al. (2011), com 16 paraplégicos por lesão medular, verificou o impacto na qualidade de vida após um período de um ano de treinamento esportivo de basquetebol em cadeira de rodas, alcançando melhoras significativas em seis dos oito domínios do questionários SF-36, além de indicar melhora geral da média de todos os participantes antes e após o período de treinamento esportivo.

A lesão medular frequentemente causa comprometimentos na habilidade de realizar as atividades diárias e limitações das funções de 
mobilidade e participação na comunidade (CHAN et al, 2007). Comumente, os indivíduos que perdem a mobilidade dos membros inferiores se tornam sedentários, mesmo se tratando de adultos jovens e ativos (NASH et al, 2005)

Neste sentido, o estudo de Silva, Oliveira e Conceição (2005) indica que a prática de atividade física em portadores de lesão medular promove melhora do consumo máximo de oxigênio, ganho de capacidade aeróbica, redução do risco de doenças cardiovasculares e de infecções respiratórias, diminuição na incidência de complicações médicas (infecções urinárias, escaras e infecções renais), redução de hospitalizações, aumento da expectativa de vida, aumento nos níveis de integração comunitária, auxílio no enfrentamento da deficiência, favorecimento da independência, melhora da autoimagem, autoestima e satisfação com a vida e diminuição na probabilidade de distúrbios psicológicos. Além disso, o esporte aumenta a força, a estabilidade de tronco e a condição de equilíbrio dos cadeirantes praticantes de basquete.

O estudo de Orsi et al. (2011) também demonstrou que a atividade física promove benefícios físicos, psicológicos e sociais à pessoa com deficiência. $O$ esporte tem um papel fundamental na reabilitação: complementa e amplia as alternativas, estimula e desenvolve os aspectos físicos, psicológicos, sociais e favorece a independência, uma maior velocidade para a locomoção e mobilidade via cadeira de rodas, alcançada mediante maior aplicação de força na propulsão.

Mello et al. (2005) acrescentam que o esporte para paraplégicos e tetraplégicos prepara o indivíduo para a vida em sociedade e para o tempo livre, oferece vivências de êxito, aumenta a tolerância à frustração, promove o contato social, torna os indivíduos mais independentes, melhora a autoimagem e a autoestima, desenvolve o potencial residual, melhora a condição orgânica e funcional, além de aprimorar as qualidades físicas como a resistência, força e velocidade.

Diante disso, considera-se que os esportes e o lazer devem ser incluídos como parte do tratamento médico para indivíduos com lesão medular, uma vez que são fundamentais no processo de enfrentamento da "desvantagem" pelos deficientes físicos. Constitui, assim, papel fundamental na reabilitação, estimulando e desenvolvendo aspectos físicos, psicológicos e sociais e, por conseguinte, favorecendo a independência funcional (HANSON; NABAVI; YUEN, 2001).

Sobre estes aspectos, Fox et al. (2000) indicam que a prática orientada de atividades físicas ou esportivas promove vários benefícios orgânicos aos portadores de deficiência física, relacionados com os aspectos metabólicos, músculo-osteoarticular e cardiorrespiratório. 
No entanto, percebeu-se que, no grupo de praticantes de basquetebol em cadeira de rodas, os domínios relacionados a dor, aspectos sociais, aspectos emocionais e saúde mental foram os mais comprometidos. Tais dificuldades e comprometimentos da qualidade de vida observados nos indivíduos com lesão medular, podem estar relacionados ao fato das modificações destes pacientes provocadas pela lesão serem irreversíveis, mesmo com o atual desenvolvimento da ciência. Assim, estes indivíduos demandam um programa de reabilitação longo e oneroso, que objetiva adaptar o indivíduo à sua nova condição (VALL; BRAGA; ALMEIDA, 2006).

\section{Conclusão}

Os resultados obtidos neste estudo permitiram concluir que os participantes lesados medulares que praticavam basquetebol em cadeira de rodas apresentaram diferenças significativas em relação aos aspectos relacionados a capacidade funcional, aspectos físicos e vitalidade.

Assim, sugere-se que o basquetebol em cadeira de rodas seja uma possível estratégia terapêutica de reabilitação ao lesado medular, uma vez que pode proporcionar benefícios físicos, psicológicos e sociais, capazes de melhorar a qualidade de vida dos indivíduos acometidos. Neste sentido, recomendam-se novos estudos com maior número de participantes para que se possam analisar os efeitos do treinamento esportivo em pessoas com lesão da medula espinhal, como também compreender as diversas variáveis do treinamento que podem influenciar a qualidade de vida destes indivíduos.

\section{Referências}

BAMPI, L. N. da S.; GUILHEM, D.; LIMA, D. D. Qualidade de vida em pessoas com lesão medular traumática: um estudo com o WHOQOLbref. Rev. Bras. Epidemiol., v. 11, n. 1, p.57-77, dez. 2008.

CICONELLI, R. M. Medidas de avaliação de qualidade de vida. Rev. Bras. Reumatologia, v. 43, n. 2, p.9-13, 2003.

CICONELLI, R. M. Tradução para a língua portuguesa e validação do questionário genérico de avaliação de qualidade de vida SF-36 (Brasil, SF-36). Rev. Bras. Reumatologia, v. 39, n. 3, 1999.

CHAN, S.C. et al. User satisfaction, community participation and quality of life among Chinese wheelchair users with spinal cord injury: a preliminary study. Occup. Ther. Int, v.14, n.3, p. 123-143, 2007. 
FOX, K. R et al.The case for exercise in the promotion of mental health and psychological well-being. In: BIDDLE, S. J. H; FOX, K. R.; BOUTCHER, S. H. Physical Activity and Psychological Well-being. London: Routledge, 2000, p.1-9.

GORLA, J. I. et al. A composição corporal em indivíduos com lesão medular praticantes de basquetebol em cadeira de rodas. Arq. Ciênc. Saúde Unipar, Umuarama, v. 11, n. 1, p. 39-44, jan./abr. 2007.

HANSON, C. S.; NABAVI, D.; YUEN, H. K. The effect of sports on level integration as reported by persons with spinal cord injury. Am J Occ Ther v. 55, n. 3, p.332-8, 2001.

HORTA, R. B. et al. Análise comparativa da composição corporal e de praticantes e não praticantes de desporto adaptado. Movimentum: Revista Digital de Educação Física - Ipatinga, v. 4, n. 1, fev./jul. 2009.

MELLO, M. T. et al. O exercício físico e os aspectos psicobiológicos. Revista Brasileira de Medicina do Esporte, v. 11, n. 3, p. 203-7, maio/ jun. 2005.

NASCIMENTO, L. G.; SILVA, S. M. L. Benefícios da atividade física sobre o sistema cardiorespiratório, como também, na qualidade de vida de portadores de lesão medular: Uma Revisão. Revista Brasileira de Prescrição e Fisiologia do Exercício, São Paulo, v. 1, n. 3, p.42-50, maio/jun. 2007.

NASH, M.S. et al. Exercise as a Health-Promotion Activity Following Spinal Cord Injury. Journal of Neurological Physical Therapy, v. 29, n.2, p.87-115, 2005.

NOCE, F.; SIMIM, M. A. M; MELLO, M. T. A percepção de qualidade de vida de pessoas portadoras de deficiência física pode ser influenciada pela prática de atividade física? Rev Bras Med Esporte, Niterói, v. 15, n. 3 , p. 174-178, June 2009.

ORSI, F. et al. O esporte na qualidade de vida de indivíduos com lesão da medula espinhal: série de casos. Rev Bras Med Esporte, v. 17, n. 4, p. 254-256, ago. 2011. 
SILVA, M. C. R. da; OLIVEIRA, R. J. de; CONCEIÇÃO, M. I. G. Efeitos da natação sobre a independência funcional de pacientes com lesão medular. Rev Bras Med Esporte, v. 11, n. 4, p. 251-256, jul./ago. 2005.

THOMAS, J. R.; NELSON, J. K.; SILVERMAN, S. J. Métodos de pesquisa em educação física. 5. ed. Porto Alegre: Artmed, 2007.

VALL, J.; BRAGA, V. A.; ALMEIDA, P. C. Estudo da qualidade de vida em pessoas com lesão medular traumática. Arq. Neuropsiquiátrico, v. 64 , n. 2, p. 451-55, fev. 2006. 